Revista Calidad en la Educación Superior

Programa de Autoevaluación Académica

Universidad Estatal a Distancia

ISSN 1659-4703

Costa Rica

revistacalidad@uned.ac.cr

\title{
LA GESTIÓN DE LA CALIDAD EN LA UNIVERSIDAD ESTATAL A DISTANCIA: Reflexiones sobre las tareas pedagógicas en una gestión hacia la calidad
}

\section{QUALITY MANAGEMENT IN THE UNIVERSIDAD ESTATAL A DISTANCIA: Reflections about pedagogical tasks in a quality management}

\author{
Mariana Torres Villalobos ${ }^{1}$, mtorrev@uned.ac.cr \\ Aurora Trujillo Cotera ${ }^{2}$, atrujillo@uned.ac.cr \\ Volumen 5, Número 1 \\ Mayo 2014 \\ pp. $223-239$
}

Recibido: enero, 2014

Aprobado: abril, 2014

1 Máster en Economía con énfasis en banca y mercado de capitales de la UCR. Licenciada y Bachiller en Dirección de

Empresas. Actualmente labora como Asesora-Investigadora del Programa de Autoevaluación de la Universidad Estatal a Distancia de Costa Rica

${ }^{2}$ Doctora en Educación, Máster en Currículo y Docencia Universitaria y Licenciada en Odontología. Investigadoraasesora destacada del Programa de Autoevaluación Académica de la Universidad Estatal a Distancia, Costa Rica. 


\section{Resumen}

La rendición de cuentas y la eficiencia en la gestión institucional es en nuestros días una nueva tarea pedagógica en las instituciones educativas, por lo que se hace evidente la necesidad de establecer mecanismos de gestión de la calidad, que den garantía pública acerca del cumplimiento de los objetivos que satisfagan las demandas de la sociedad. Conceptualizar una gestión de calidad significa estructurarla para el cumplimiento de estándares bajo los cuales se enrumbe el quehacer de una institución educativa.

Palabras claves: gestión, calidad y mejoramiento continuo.

\section{Abstract}

Rendering accounts and efficiency in institutional management is nowadays a new teaching job in educational institutions. So that, the need to establish mechanisms for quality management, to give public assurance about the achievement of the objectives is evident that meet the demands of society. Quality management structure means to comply with standards under which the work of an educational institution will bring a new direction.

Keywords: management, quality and continuous improvement.

\section{Introducción}

La educación superior en las últimas décadas se ha convertido en un importante factor de cambio social y de crecimiento económico para la realización de objetivos colectivos de la sociedad (OECD, 2008), así como es un indicador de crecimiento y desarrollo de los países. Con base en lo anterior, hoy un tema central en las políticas públicas educativas, debido a su rápido crecimiento y a la diversificación de la población estudiantil, son las nuevas tareas pedagógicas que conllevan la rendición cuentas y la eficiencia en la gestión institucional.

Estas nuevas tareas pedagógicas están dirigidas a construir una educación superior o terciaria en constante diálogo de la comunidad académica con el mercado laboral, que se pueda permear de la experiencia de los estudiantes, por 
medio de ambientes adecuados de interacción, en la cual la currícula responda a los requerimientos económicos, sociales y productivos del entorno. Asimismo es necesario que se implemente la investigación y extensión; aunado a una evaluación del logro en procura de revisión constante de la gestión realizada, la cual debe ser de calidad.

Por lo que, es evidente la necesidad de establecer mecanismos de gestión de la calidad, que den garantía pública acerca del cumplimiento de los objetivos declarados; brinde información pertinente y oportuna para la toma de decisiones; que satisfagan las demandas de la sociedad. Conceptualizar una gestión de calidad significa estructurarla bajo la definición de estándares en los cuales se enrumbe el quehacer de una institución educativa. Por esta razón, estos parámetros conceptualizados como estándares debes ser consensuados por quienes serán evaluados, con la finalidad de que no sean laxos ni punitivos.

Como se ha visto, según Águila (2005) "la calidad de la educación universitaria no se entiende, ni se mide ni se hace como hace medio siglo atrás", con lo que se está de acuerdo en el sentido de que el mundo está en constante cambio, lo que repercute en la calidad de la educación superior y por ende en la sociedad misma. Estos cambios en la educación han sido provocados por procesos de globalización, desarrollo económico y tecnológico, como la implementación de plataformas educativas digitales y otras tecnologías de la información.

Con relación a ese cambio y siguiendo la idea de Águila (2005) el esquema de calidad universitaria debe contemplar aspectos tan relevantes como la pertinencia social, las exigencias y normas internacionales más generalizadas y la comparación con estándares establecidos". (p.2, 3).

En los programas académicos de las universidades latinoamericanas por to general no se desarrollan evaluaciones de manera permanente, sino que impera 
un continuismo autocrático renuente al cambio y a la rendición de cuentas con respecto a la pertinencia, congruencia y actualización de los programas y servicios académicos. A este respecto según Cardoso, Cerecedo y Ramos (2011) establece:

La calidad educativa se entiende como la síntesis de atributos que posee una institución o programa educativo y que se encuentra determinada por la capacidad que tienen las instituciones para preparar al individuo, de tal manera que pueda adaptarse y contribuir con el crecimiento, desarrollo económico y social mediante su incorporación al mercado laboral (p.70).

Para la UNESCO, la gestión de la calidad tiene como propósitos: el control, la rendición de cuentas y la mejora continua. Estos se relacionan con criterios mínimos de calidad que cada institución de educación superior establecen o asumen, identificando a nivel interno fortalezas y debilidades, y externo oportunidades y amenazas, que en conjunto determinan un plan de mejoramiento en pro de la calidad de la oferta académica.

El éxito de la gestión de una institución educativa, en este caso las universidades, depende en gran medida de la forma en la cual se realice su dirección y control, para que sea sistemático y transparente el proceso, enfocando su desempeño en el mejoramiento continuo.

La gestión de la calidad denominada también como sistema de gestión de la calidad, es aquel conjunto de normas correspondientes a una organización, vinculadas entre sí y a partir de las cuales es que la empresa u organización en cuestión podrá administrar de manera organizada la calidad de la misma. La misión siempre estará enfocada hacia la mejora continua. 


\section{Evolución histórica del concepto de Calidad}

La evolución de la gestión de la calidad ha sido ampliamente analizada desde el punto de vista académico, de esta forma ha transcurrido a través de cuatro etapas progresivas: la inspección, el control, el aseguramiento y la gestión de la calidad total como se muestra a continuación en la tabla 1 (Camisón, C. et al., 2007, p.147).

\section{Tabla 1. Conceptos de calidad}

\begin{tabular}{|c|c|c|c|}
\hline Autores & Enfoque & Acento diferencial & Desarrollo \\
\hline Platón & Excelencia & $\begin{array}{l}\text { Calidad absoluta } \\
\text { (producto) }\end{array}$ & $\begin{array}{l}\text { Excelencia como superioridad absoluta "lo mejor". } \\
\text { Asimilación con el concepto de lujo } \\
\text { Analogía con la calidad del diseño. }\end{array}$ \\
\hline $\begin{array}{l}\text { Shewhart } \\
\text { Crosby }\end{array}$ & $\begin{array}{l}\text { Técnico: } \\
\text { conformidad con } \\
\text { especificaciones }\end{array}$ & $\begin{array}{l}\text { Calidad comprobada / } \\
\text { Controlada (procesos) }\end{array}$ & $\begin{array}{l}\text { Establecer especificaciones. } \\
\text { Medir la calidad por la proximidad real a los } \\
\text { estándares. } \\
\text { Énfasis en la calidad de conformidad. Cero } \\
\text { defectos. }\end{array}$ \\
\hline $\begin{array}{l}\text { Deming, } \\
\text { Taguchi }\end{array}$ & $\begin{array}{l}\text { Estadístico: } \\
\text { pérdidas } \\
\text { mínimas para la } \\
\text { sociedad, } \\
\text { reduciendo la } \\
\text { variabilidad y } \\
\text { mejoramiento } \\
\text { de estándares. }\end{array}$ & $\begin{array}{l}\text { Calidad generada } \\
\text { (producto y procesos) }\end{array}$ & $\begin{array}{l}\text { La calidad es inseparable de la eficacia } \\
\text { económica. } \\
\text { Un grado predecible de uniformidad y fiabilidad a } \\
\text { bajo costo. } \\
\text { La calidad exige disminuir la variabilidad de las } \\
\text { características del producto alrededor de los } \\
\text { estándares y su mejora permanente. } \\
\text { Optimizar la calidad del diseño para mejorar la } \\
\text { calidad de conformidad. }\end{array}$ \\
\hline $\begin{array}{l}\text { Feigenba } \\
\text { um Juran } \\
\text { Ishikawa }\end{array}$ & $\begin{array}{l}\text { Aptitud para el } \\
\text { uso. }\end{array}$ & $\begin{array}{l}\text { Calidad } \\
\text { (sistema) }\end{array}$ & $\begin{array}{l}\text { Traducir las necesidades de los clientes en las } \\
\text { especificaciones. } \\
\text { La calidad se mide por lograr la aptitud deseada } \\
\text { por el cliente. } \\
\text { Énfasis tanto en la calidad del diseño como de } \\
\text { conformidad. }\end{array}$ \\
\hline $\begin{array}{l}\text { Parasura } \\
\text { man } \\
\text { Berry } \\
\text { Zeithaml }\end{array}$ & $\begin{array}{l}\text { Satisfacción de } \\
\text { expectativas del } \\
\text { cliente }\end{array}$ & $\begin{array}{ll}\begin{array}{l}\text { Calidad } \\
\text { (servicio) }\end{array} & \text { satisfecha }\end{array}$ & $\begin{array}{l}\text { Alcanzar o superar las expectativas de los } \\
\text { clientes. } \\
\text { Énfasis en la calidad del servicio. }\end{array}$ \\
\hline $\begin{array}{l}\text { Evans } \\
\text { (Procter } \\
\& \\
\text { Gamble) }\end{array}$ & Calidad total & $\begin{array}{l}\text { Calidad gestionada } \\
\text { (empresa y su sistema } \\
\text { de valor) }\end{array}$ & $\begin{array}{l}\text { Calidad significa crear valor para los grupos de } \\
\text { interés. } \\
\text { Énfasis en la calidad en toda la cadena y el } \\
\text { sistema de valor. }\end{array}$ \\
\hline
\end{tabular}

Fuente: Camisón, Cruz y González, 2007 
El concepto de gestión de la calidad para procesos de autoevaluación y acreditación de carreras y programas de grado y posgrado, según Cardoso y Cerecedo (2011) debe ser operacional, convertir en elementos fácilmente manejables dentro una guía, modelo o procedimiento, que debe abarcar de una $u$ otra forma las funciones sustantivas del programa, llevando implícito el concepto de evaluación y que esté ligado a la pertinencia social.

\section{Los sistemas de gestión de la calidad}

La Norma ISO 9000:2005 define los sistemas de gestión de calidad son concebidos como aquellas parte del sistema de gestión de la organización que se enfoca en el logro de resultados, en relación con los objetivos de la calidad, para satisfacer las necesidades, expectativas y requerimientos de las partes interesadas.

Estos sistemas permiten a las organizaciones, según esta norma lo siguiente:

$\checkmark$ Identificar sus fortalezas y debilidades.

$\checkmark$ Evaluar con base en modelos genéricos,

$\checkmark$ Proporcionar una base para la mejora continua, y

$\checkmark$ Comprensión del entorno.

La gestión de la calidad según la Norma ISO (2005) cuenta con ocho principios que pueden ser utilizados por las autoridades con el fin de conducir a la organización hacia una mejora en el desempeño:

1. Enfoque al usuario.

2. Liderazgo.

3. Participación del personal.

4. Enfoque basado en procesos.

5. Enfoque de sistema para la gestión.

6. Mejora continua.

7. Enfoque basado en hechos para la toma de decisión.

8. Relaciones mutuamente beneficiosas con el proveedor. 


\section{¿Por qué es importante evaluar la gestión de la calidad en educación?}

Cardoso y Cerecedo (2011) "establecen que los niveles de desarrollo y crecimiento de una nación están sustentados por la calidad de la educación, ya que es reconocida como la base del desarrollo, como elemento de innovación y como ventaja estratégica para la producción"(p. 81).

Las instituciones de educación superior deben enfocarse en ofrecer programas educativos pertinentes a la sociedad de manera eficiente y eficaz, y poder demostrarlo a través de certificaciones o acreditaciones, ya que éste será una estrategia de las autoridades a la hora de realizar la asignación de los recursos económicos, los cuales pueden dependen del desarrollo de las actividades productivas del país. Además la entrada de nuevas universidades al mercado, y el surgimiento de rankings que las califican provocan un aumento en la competencia.

\section{La nueva Visión de la Educación Superior Universitaria Estatal Costarricense}

En las últimas tres décadas, se ha encontrado en la literatura una preocupación permanente por la evaluación de la calidad universitaria, así como también de la calidad de las instituciones y de los programas académicos, respondiendo esta propuesta educativa a las demandas y necesidades sociales actuales. En las universidades los programas académicos no se han evaluado de manera continua, sino que se han mantenido igual en el tiempo dejando de lado la función real de la evaluación: el mejoramiento continuo.

Vázquez L. (2005) sostiene que:

Emerge un patrón de evaluación en las instituciones de educación superior, que les permite ser capaces de:

$\checkmark$ Alcanzar el objetivo deseado.

$\checkmark$ Realizar una buena conexión entre el entorno y los recursos de una organización y competencia; debe ser factible y apropiada. 
$\checkmark$ Ser capaz de proporcionar a la organización una ventaja competitiva; debería ser única y mantenible en el tiempo.

$\checkmark$ Ser dinámico, flexible y capaz de adaptarse a las situaciones cambiantes. (párrafo 6)

La educación superior y en especial las universidades públicas comparten una responsabilidad social que lleva implícito cada día más la rendición de cuenta orientada a la excelencia académica y el interés de ofertar carreras de calidad.

En Costa Rica, producto del libre mercado en el campo de la educación superior y la preocupación nacional e internacional por la calidad de los mismos, se crea mediante la Ley 8256 en mayo del 2002 el Sistema Nacional de Acreditación de la Educación Superior (SINAES). Este se crea por convenio entre las instituciones de educación superior universitaria estatal, al cual podrán adherirse las instituciones de educación superior universitaria privada, el cual se incorpora como órgano adscrito al Consejo Nacional de Rectores.

Trujillo (2012) refiere que en el Plan Nacional de la Educación Superior 20112015, se señala el eje de Calidad del Desarrollo Sistémico, como acción inmediata la Profundización del Desarrollo de la Cultura de Acreditación de las Carreras y Programas. Deben desarrollar estrategias integrales que les permitan evaluar, actualizar y reorganizar su quehacer académico, a la luz de la misión histórica y las tendencias del desarrollo nacional, regional e internacional.

En lo que concierne a la evaluación académica universitaria se contempla: la autoevaluación y acreditación de carreras y programas académicos y en un mediano plazo la evaluación institucional de los servicios que apoyan a la academia.

Las actividades académicas del SINAES son de interés público, con la finalidad de planificar, organizar, desarrollar, implementar, controlar y dar seguimiento a un proceso de acreditación que garantice continuamente la calidad de las carreras, 
los planes y programas ofrecidos por las instituciones de educación superior, y salvaguarde la confidencialidad del manejo de los datos de cada institución.

Durante su gestión, al SINAES se han adherido 19 de las 63 universidades existentes en el país, de las cuales han acreditado 74 carreras universitarias, 36 se han reacreditado, y cuatro por segunda vez. Han fomentado las investigaciones en este campo y sus procesos de gestión de calidad han sido acreditados a su vez por el Consejo Centroamericano de Acreditación de la Educación Superior (CCA) por cinco años, organismo formalmente reconocido por todos los países del Istmo. y es la cuarta agencia de todo el mundo, la primera de América Latina, en recibir el reconocimiento de calidad de la Red Internacional de Agencias de Aseguramiento de la Calidad de la Educación Superior, INQAAHE, que es la más importante red internacional de aseguramiento en la calidad educativa del planeta.

\section{Construyendo un sistema de gestión de la calidad en la Universidad Estatal a Distancia}

La UNED está anuente a "considerar la gestión de la calidad académico administrativa como el reto permanente por conseguir los objetivos propuestos, aportando los medios necesarios para lograrlo" Acuña, Cox, Elizondo y Jiménez (2013) fomentando una cultura de calidad que posibilite evaluar las condiciones y características de los resultados y servicios obtenidos en la institución. (p.26).

Esta institución deja claramente plasmada en sus lineamientos de política institucional guías claves enfocadas en este compromiso. Para lograrlo promueve el mejoramiento continuo por medio de la evaluación y autoevaluación, factores determinantes en la toma de decisiones, la rendición de cuentas y el logro de la mejora continua, como una práctica cotidiana.

En esta línea el gestionar la calidad en la institución ha sido un proceso de crecimiento y maduración iniciada con acciones de autoevaluación, evaluación 
externa con fines de acreditación, elaboración de planes de mejora y seguimiento de los mismos de carreras y programas de grado y posgrado. Estas prácticas al pasar el tiempo se convierten en insumos al metaevaluar, que posibilitan fortalecer prácticas exitosas, corregir el rumbo por seguir y adoptar nuevas prácticas e incursionar en acciones y proyectos de investigación, producción académica y desarrollo profesional.

Es por ello, que el Consejo Universitario en la sesión N 1234 - 2002, Artículo III, celebrada el 2 de abril del 2002 crea el Programa de Autoevaluación Académica (PAA) como unidad estratégica dirigida a la autoevaluación de las programas de pregrado, grado y posgrado para fines de acreditación.

Desde su creación, el PAA se ha dedicado al apoyo y la asesoría de las carreras en:

$\checkmark$ Recolección de Información de académicos, estudiantes, administrativos, autoridades, graduados y empleadores.

$\checkmark$ Recolección de información sobre estado de servicios que ofrece la carrera.

$\checkmark$ Revisión del accionar de la carrera con base en la actualidad, congruencia y pertinencia de cada asignatura que conforma el Plan de Estudios.

$\checkmark$ Seguimiento de las actividades realizadas por la carrera en los últimos cuatro años.

$\checkmark$ Asesoría y acompañamiento en la elaboración de informes de autoevaluación y de avance de cumplimiento de Compromiso de Mejoramiento

$\checkmark$ Acompañamiento en la elaboración de los Planes de Gestión Académica.

A través de estos doce años de funcionamiento, el PAA trabaja en conjunto con las Escuelas en la autoevaluación de carreras y programa de grado y posgrado, ya sea para acreditación o mejoramiento. Como se indica en las tablas 2, 3 y 4 en este momento es la situación de la institución 
Tabla 2. Distribución de carreras acreditadas por SINAES según Escuelas de la UNED

\begin{tabular}{|c|c|c|c|c|c|c|c|}
\hline \multirow[b]{2}{*}{ Escuela } & \multirow[b]{2}{*}{ Carrera } & \multicolumn{4}{|c|}{$\begin{array}{c}\text { Grado } \\
\text { Académico }\end{array}$} & \multirow[b]{2}{*}{$\begin{array}{l}\text { Tiempo de } \\
\text { acreditación }\end{array}$} & \multirow[b]{2}{*}{$\begin{array}{l}\text { Número de } \\
\text { acreditación }\end{array}$} \\
\hline & & $\begin{array}{l}\text { 믐 } \\
\text { 흥 } \\
3 \\
\text { 응 }\end{array}$ & 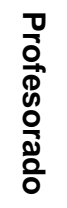 & 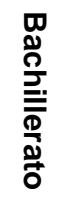 & 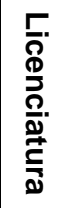 & & \\
\hline \multirow{3}{*}{$\begin{array}{l}\text { Ciencias de la } \\
\text { Educación }\end{array}$} & Informática Educativa* & $x$ & & $x$ & $x$ & 4 años & 1 \\
\hline & Educación Preescolar & $x$ & & $x$ & $x$ & 4 años & 1 \\
\hline & Administración Educativa & & & $\mathrm{X}$ & $\mathrm{X}$ & 4 años & 1 \\
\hline \multirow{3}{*}{$\begin{array}{l}\text { Ciencias } \\
\text { Exactas y } \\
\text { Naturales }\end{array}$} & Enseñanza de la Matemática* & & $\mathrm{x}$ & $\mathrm{X}$ & & 4 años & 2 \\
\hline & Enseñanza de las Ciencias Naturales & & $\mathrm{x}$ & $\mathrm{x}$ & & 4 años & 1 \\
\hline & Manejo de los Recursos Naturales & & & $x$ & $x$ & 4 años & 1 \\
\hline \multirow{7}{*}{$\begin{array}{l}\text { Ciencias de la } \\
\text { Administración }\end{array}$} & $\begin{array}{c}\text { Administración de Empresas con } \\
\text { énfasis en Contaduría }\end{array}$ & & $x$ & $x$ & & 6 años & 1 \\
\hline & Administración de Empresas & $x$ & & & & \multirow{6}{*}{4 años } & \multirow{6}{*}{1} \\
\hline & Énfasis en Banca y Finanzas & & $\mathrm{x}$ & $\mathrm{x}$ & & & \\
\hline & Énfasis en Dirección de Empresas & & $\mathrm{x}$ & $\mathrm{x}$ & & & \\
\hline & Énfasis en Recursos Humanos & & $x$ & $\mathrm{x}$ & & & \\
\hline & Énfasis en Producción & & $\mathrm{x}$ & $\mathrm{x}$ & & & \\
\hline & Énfasis en Mercadeo & & $x$ & $x$ & & & \\
\hline
\end{tabular}

Fuente: Programa de Autoevaluación Académica, 2014

* Estas carreras continúan acreditadas pues están a la espera de visita de pares para su reacreditación. 
Tabla 3. Distribución de carreras en procesos de autoevaluación con miras a acreditación por parte del SINAES según Escuelas de la UNED

\begin{tabular}{|c|c|c|c|c|c|c|c|}
\hline \multirow[b]{2}{*}{ Escuela } & \multirow[b]{2}{*}{ Carrera } & \multicolumn{4}{|c|}{ Títulos } & \multirow[b]{2}{*}{$\begin{array}{c}\text { Fecha que } \\
\text { presentó } \\
\text { informe a } \\
\text { SINAES }\end{array}$} & \multirow[b]{2}{*}{$\begin{array}{c}\text { Agencia } \\
\text { Acreditadora }\end{array}$} \\
\hline & & 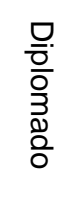 & 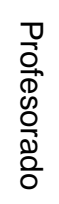 & 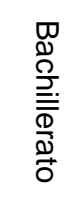 & 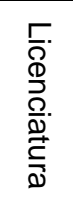 & & \\
\hline \multirow{4}{*}{$\begin{array}{l}\text { Ciencias de } \\
\text { la } \\
\text { Educación }\end{array}$} & & & & & & & \multirow{3}{*}{ SINAES } \\
\hline & Educación Especial & & & $X$ & & 2010 & \\
\hline & Enseñanza Básica en I y II ciclo & $x$ & & $x$ & $x$ & 2010 & \\
\hline & $\begin{array}{c}\text { Educación Preescolar: } \\
\text { estimulación y corrección del } \\
\text { lenguaje }\end{array}$ & & & & $x$ & 2012 & SINAES \\
\hline \multirow{2}{*}{$\begin{array}{l}\text { Ciencias } \\
\text { Sociales y } \\
\text { Humanidad } \\
\text { es }\end{array}$} & $\begin{array}{c}\text { Enseñanza del Inglés para primer } \\
\text { y segundo ciclo }\end{array}$ & $x$ & & $x$ & $x$ & $26 / 4 / 2013$ & \\
\hline & Gestión Turística Sostenible & $\mathrm{X}$ & & $\mathrm{X}$ & & $09 / 10 / 2013$ & \\
\hline
\end{tabular}

Fuente: Programa de Autoevaluación Académica, 2014 
Tabla 4. Distribución de carreras en procesos de autoevaluación por parte del SINAES según Escuelas de la UNED

\begin{tabular}{|c|c|c|c|c|c|c|}
\hline \multirow[t]{2}{*}{ Escuela } & \multirow[t]{2}{*}{ Carrera } & \multicolumn{4}{|c|}{ Titulo } & \multirow{2}{*}{$\begin{array}{c}\text { Año que } \\
\text { inicio } \\
\text { proceso }\end{array}$} \\
\hline & & Dip & Prof & Bach & Lic & \\
\hline \multirow{6}{*}{$\begin{array}{c}\text { Ciencias Exactas y } \\
\text { Naturales }\end{array}$} & Ingeniería Agronómica & & & $\mathrm{x}$ & $\mathrm{x}$ & 2012 \\
\hline & Ingeniería Agroindustrial & & & $\mathrm{x}$ & $x$ & 2012 \\
\hline & Diplomado en Informática & $\mathrm{x}$ & & & & 2012 \\
\hline & $\begin{array}{l}\text { Bachillerato en Ingeniería } \\
\text { Informática }\end{array}$ & & & $x$ & & \\
\hline & $\begin{array}{c}\text { Licenciatura en Ingeniería } \\
\text { Informática }\end{array}$ & & & & $\mathrm{x}$ & 2012 \\
\hline & $\begin{array}{l}\text { Licenciatura en Ingeniería } \\
\text { Informática con énfasis en: } \\
\text { - Administración de Proyectos } \\
\text { - Calidad de Software } \\
\text { - Desarrollo de aplicaciones en } \\
\text { web }\end{array}$ & & & & $x$ & 2012 \\
\hline
\end{tabular}

Fuente: Programa de Autoevaluación Académica, 2014 
Como se observa en las tablas anteriores, las autoridades, Escuelas, Carreras y Programas, dependencias de apoyo y en general toda la universidad, incluyendo a estudiantes, graduados con el apoyo de los empleadores, se encuentran comprometidas con estos procesos de evaluación con el propósito fundamental del mejoramiento continuo. Además, otro aspecto en que se trabaja es en crear una propuesta para la evaluación de las cátedras, así como del Programa de Estudios Generales, pues al autorregular tanto las cátedras como la formación humanística, se benefician todas las carreras y programas de la institución.

Apoyando estos procesos y con el fin de promocionar una cultura de calidad en la comunidad universitaria, el PAA ha considerado relevante la publicación de la Revista de Calidad en la Educación Superior, creada desde el 2010 y que cuenta con dos ediciones anuales, con el fin de colaborar con la difusión de la calidad en la gestión académica universitaria. Este esfuerzo se ha visto reflejado en indexaciones en Latindex, Dialnet y E-revistas.

También el Programa de Autoevaluación Académica trabaja en articulación con Escuelas y Dependencias de la UNED, fortaleciendo procesos de docencia, investigación y extensión y a su vez consolidándose a sí mismo.

\section{Conclusión: desafíos para una adecuada gestión de la calidad en la UNED}

La implementación de un sistema de gestión de la calidad, ha exigido la realización de una serie de actividades que involucran a la universidad como un todo, desde la definición de la política de la institución, hasta la aplicación de los controles de calidad que exige el sistema.

Con base en los aspectos teóricos estudiados anteriormente, es importante destacar como conclusión, los desafíos más importantes que debe solventar la UNED para lograr una de adecuada gestión de calidad en esta institución educativa, los cuales se citan a continuación: 
$\checkmark$ Congruencia, pertinencia y actualidad en los programas académicos. Es necesario realizar un análisis periódico sobre las nuevas tendencias en las áreas de estudio y la pertinencia de los programas en la actualidad económica del país.

$\checkmark$ Integración de la docencia, investigación y extensión en la jornada de los docentes. Para lograr esta integración es necesario contar con personal académico que cuente con una carga académica adecuada para su desarrollo en estas tres áreas.

$\checkmark$ Autoevaluación, Autorregulación y Mejoramiento de las carreras y Escuelas. Cada carrera corresponde a una disciplina científica diferente, por lo tanto sus debilidades y fortalezas son muy particulares y conocidas por sus actores. De la misma forma, las escuelas conjuntan estas disciplinas las cuales desde una perspectiva académica pueden buscar sus vías de solución.

$\checkmark$ El acompañamiento de la gestión institucional. La gestión académica va de la mano de la gestión administrativa, una y otra deben estar orientadas al cumplimiento de los mismos objetivos de la gestión de la calidad.

$\checkmark$ Reestructuración organizacional en la búsqueda de una gestión eficaz. Los procesos de autoevaluación deben llevar a cambios, las mejoras deben llevarse del papel a los hechos con la finalidad de que el cambio sea no únicamente cosmético sino que apunte a la adopción de una nueva cultura organizacional.

$\checkmark$ Generar y divulgar información sobre el desempeño de la institución. La rendición de cuentas es una obligación de toda institución educativa, sobre todo de carácter público, por lo que el mecanismo ideal para llevarla a cabo es la autoevaluación que conlleve a una evaluación externa que permita optimizar el desempeño de la gestión institucional.

$\checkmark$ Otro aspecto en que se trabaja es en crear una propuesta para la evaluación de las cátedras, así como del Programa de Estudios Generales. 
$\checkmark$ Promover la innovación tecnológica en los procesos de gestión de la calidad en procura de su agilización, flexibilización y optimación de resultados.

A la Universidad Estatal a Distancia es mucho lo que le queda por hacer para consolidar una cultura que consolide la gestión de la calidad, vendrán logros, retos y desafíos. Sin embargo el entusiasmo y la sensibilización de los colaboradores se observa cada día más y se enfoca en asegurar la sostenibilidad del trabajo realizado.

La gestión se ha convertido en un aprendizaje continuo y de constante crecimiento, consolidándose el trabajo en equipo; tarea fundamental en estos procesos que requieren ser críticos, reflexivos, analíticos y participativos, pero también que ofrecen una oportunidad para destacar las fortalezas de los programas y la institución, orientándonos también en establecer la coherencia entre la misión y los proyectos que se ofrecen a la sociedad interpretando sus necesidades.

\section{Referencias Bibliográficas}

Acuña, J., Cox, J., Elizondo, F. y Jiménez, L. (2013.) Universidades costarricenses: pensando y construyendo juntas. CONARE - UNIRE. Revista Electrónica Calidad en la Educación Superior (4)2. Recuperado de http://web.uned.ac.cr/revistas/index.php/caes/article/view/154/172

Águila, V. (2005) El concepto calidad en la educación universitaria: clave para el logro de la competitividad institucional Revista Iberoamericana de Educación 35-5 Recuperado de http://www.rieoei.org/deloslectores/880Aguila.PDF

Camisón, C., Cruz, S. y González, T. (2007). Gestión de la Calidad. Madrid: Pearson/Prentise Hall. 
Cardoso, E. y Cerecedo, M. (2011). Propuesta de indicadores para evaluar la calidad de un programa de posgrado en Educación. Revista electrónica de Innovación Educativa 13(2), 68-82. Recuperado de http://redie.uabc.mx/vol13no2/contenido-cardosocerecedo.html

Cardoso, E., Cerecedo, M. y Ramos, J. (2013). Evaluación institucional basada en los sistemas suaves. Recuperado de http://www.barnesandnoble.com/sample/read/9781463361822

CONARE- OPES (2011) Plan Nacional de la Educación Superior 2011 - 2015. San José, Costa Rica.

Norma Internacional ISO 9000. (2005). Sistemas de gestión de la calidad. Fundamentos y vocabulario. Suiza: Ginebra.

UNESCO. (s.f.). Aseguramiento de la calidad en la educación superior. Recuperado de http://www.iiep.unesco.org/es/focus-on-highereducation/quality-assurance-in-higher-education.html

Scheele, J. (2008). El aseguramiento de la calidad en la educación terciaria no universitaria. OECD. Recuperado de http://www.cpce.cl/

Trujillo, A. (2012). Presentación sobre lineamientos conceptuales y procedimientos generales sobre la Autoevaluación en la Universidad Estatal a Distancia en procura de la Gestión de la calidad. Programa de Autoevaluación Académica. UNED.

Vázquez L. (2011) La "prospectiva-planificación estratégica", la garantía de calidad académica y la evaluación. Recuperado de http://arualvazquez.blogspot.com/2011/04/la-evaluacion-de-la-educacionsuperior.html 